\title{
Degeneration of the Superintegrable System with Potentials Described by the Sixth Painlevé Transcendents
}

\author{
Yoshikatsu Sasaki \\ Department of Mathematics, Hiroshima University, Higashi-Hiroshima, Japan \\ Email: sasakiyo@hiroshima-u.ac.jp
}

Received July 2014

\section{Abstract}

This article concerns the quantum superintegrable system obtained by Tremblay and Winternitz, which allows the separation of variables in polar coordinates and possesses three conserved quantities with the potential described by the sixth Painleve equation. The degeneration procedure from the sixth Painlvé equation to the fifth one yields another new superintegrable system; however, the Hermitian nature is broken.

\section{Keywords}

\section{Superintegrable System, Painlevé Equation, Degeneration}

\section{Introduction}

\subsection{Superintegrable Systems Separating in Polar Coordinates}

Consider the quantum superintegrable system which allows the separation of variables in polar coordinates and possesses three conserved quantities as follows:

$$
\begin{aligned}
& H=\frac{1}{2}\left(p_{1}^{2}+p_{2}^{2}\right)+V(r, \theta), \\
& X=L_{3}^{2}+2 S(\theta), \\
& Y=\sum_{k+l+m=3} A_{k l m}\left\{L_{3}^{k}, p_{1}^{l} p_{2}^{m}\right\}+\left\{g_{1}(x), p_{1}\right\}+\left\{g_{2}(x), p_{2}\right\},
\end{aligned}
$$

where $\boldsymbol{x}=\left(x_{1}, x_{2}\right)=(r \cos \theta, r \sin \theta), \quad p_{k}=-i \hbar \partial / \partial x_{k}(j=1,2), \quad L_{3}=x_{1} p_{2}-x_{2} p_{1}=-i \hbar \partial / \partial \theta$, $V(r, \theta)=R(r)+S(\theta) / r^{2},\{A, B\}=A B+B A$. Here $R(r), S(\theta), g_{1}(\boldsymbol{x}), g_{2}(\boldsymbol{x})$ are arbitrary functions, and $A_{k l m}$ 's are real constants. Note that the Hermitian nature of the operators causes the anti-commutator $\{$, and the parity. In the followings, we use such the notation as $\partial_{\theta}=\partial / \partial \theta$ for brevity.

Tremblay and Winternitz [1] classified the cases where the above system is superintegrable, i.e. it allows the 
third conserved quantity $Y$, and obtained $S(\theta)$ which is written by the solution of the sixth Painlevé equation. If $V=S(\theta) / r^{2}, T=T(\theta), T^{\prime}=S(\theta)$,

$$
\begin{aligned}
& H=-\frac{\hbar^{2}}{2}\left\{\partial_{r}^{2}+r^{-1} \partial_{r}+r^{-2} \partial_{\theta}^{2}\right\}+S(\theta) / r^{2}, \\
& X=-\hbar^{2} \partial_{\theta}^{2}+2 S(\theta), \\
& Y=\left\{L_{3}^{2}, p_{1}\right\}+\left\{G_{1} \cos \theta-G_{2} r \sin \theta, p_{1}\right\}+\left\{G_{1} \sin \theta+G_{2} r \cos \theta, p_{2}\right\} \\
& \quad=-\hbar^{2}\left\{\partial_{\theta}^{2}, \cos \theta \partial_{r}-\frac{1}{r} \sin \theta \partial_{\theta}\right\}+\left(\left\{\frac{1}{r} G_{1}, \partial_{r}\right\}+\left\{G_{2}, \frac{1}{r} \partial_{\theta}\right\}\right) r, \\
& G_{1}=G_{1}(r, \theta)=\beta(\theta), \\
& G_{2}=G_{2}(r, \theta)=\frac{1}{r} \beta^{\prime}(\theta)-2 S^{\prime}(\theta) \cos \theta, \\
& \beta(\theta)=\beta_{0}(\theta)+\left(-T \sin \theta+2 T^{\prime} \cos \theta\right), \\
& \beta_{0}(\theta)=\beta_{1} \cos \theta+\beta_{2} \sin \theta ; \beta_{1}, \beta_{2}: \text { const. }
\end{aligned}
$$

The commutation $[H, Y]=0$ is reduced to

$$
\begin{aligned}
& \hbar^{2}\left(-T^{\prime \prime \prime \prime} \sin \theta-4 T^{\prime \prime \prime} \cos \theta+6 T^{\prime \prime} \sin \theta+4 T^{\prime} \cos \theta\right)+4 T^{\prime \prime}\left\{3 T^{\prime} \sin \theta+T \cos \theta-\beta_{0}{ }^{\prime}(\theta)\right\} \\
& \quad+8 T^{\prime}\left\{2 T^{\prime} \cos \theta-T \sin \theta+\beta_{0}(\theta)\right\}=0 .
\end{aligned}
$$

By change of variables $(\theta, T(\theta)) \mapsto(t, w(t))$ s.t.

$$
\tan \theta=2 \sqrt{t(1-t)} /(1-2 t), \quad T=\beta_{2}+\left\{\hbar^{2} w+\frac{1}{8}\left(\hbar^{2}+4 \beta_{1}\right)(1-2 t)\right\} / \sqrt{t(1-t)},
$$

the above equation is reduced to F-VII $\left(-\frac{1}{4}-2 \beta_{1} / \hbar^{2}, 0\right)$. Here, F-VII $\left(A_{0}, A_{2}\right)$ is a 4 th order ODE defined by

$$
\begin{aligned}
& -2 t^{2}(t-1)^{2} w^{\prime \prime \prime \prime}-6 t(2 t-1)(t-1) w^{\prime \prime \prime}-24 t(t-1) w^{\prime} w^{\prime \prime}+8(2 t-1) w w^{\prime \prime}+\left\{2 A_{0}-2-12 t(t-1)\right\} w^{\prime \prime}-4(2 t-1) w^{\prime 2} \\
& +8 w w^{\prime}+A_{2}=0,
\end{aligned}
$$

with an independent variable $t$, a dependent variable $w=w(t)$, constant parameters $A_{0}$ and $A_{2}$ (See [1] [2]). This equation can be integrated twice, and reduced to SD-I.a [1] [3] [4] with constants of integration $B_{3}$ and $A_{4}$ :

$$
-t^{2}(t-1)^{2} w^{\prime \prime 2}-4 w^{\prime}\left(t w^{\prime}-w\right)^{2}+4 w^{\prime 2}\left(t w^{\prime}-w\right)+A_{0} w^{\prime 2}+A_{2}\left(t w^{\prime}-w\right)+B_{3} w^{\prime \prime}+A_{4}=0 .
$$

Then, by the Bäcklund correspondence

$$
\begin{aligned}
w(t)= & \left\{t^{2}(t-1)^{2} / 4 u(u-1)(u-t)\right\}\left\{u^{\prime}-u(u-1) / t(t-1)\right\}^{2}+\frac{1}{8} \Theta_{\infty}^{2}(1-2 u)+\frac{1}{8} \theta_{0}^{2}(1-2 t / u) \\
& -\frac{1}{8} \theta_{1}^{2}\{1-2(t-1) /(u-1)\}+\frac{1}{8} \theta_{t}^{2}\{1-2 t(u-1) /(u-t)\}, \\
w^{\prime}(t)= & -\{t(t-1) / 4 u(u-1)\}\left\{u^{\prime} \pm \theta_{\infty} u(u-1) / t(t-1)\right\}^{2}+\frac{1}{4} \theta_{0}^{2}(u-t) / u(t-1)-\frac{1}{4} \theta_{1}^{2}(u-t) / t(u-1),
\end{aligned}
$$

SD-I.a is reduced to the sixth Painlevé equation [3]:

$$
\begin{aligned}
u^{\prime \prime}= & \frac{1}{2}\{1 / u+1 /(u-1)+1 /(u-t)\} u^{\prime 2}-\{1 / t+1 /(t-1)+1 /(u-t)\} u^{\prime} \\
& +\left\{u(u-1)(u-t) / t^{2}(t-1)^{2}\right\}\left[\theta_{\infty}^{2} / 2-\theta_{0}^{2} t / 2 u^{2}+\theta_{1}^{2}(t-1) / 2(u-1)^{2}-\left(\theta_{t}^{2}-1\right) t(t-1) / 2(u-t)^{2}\right],
\end{aligned}
$$


where the correspondence of the parameters is given by $\Theta_{\infty}=\theta_{\infty}+1$,

$$
\begin{aligned}
& A_{0}=\left(\Theta_{\infty}^{2}+\theta_{0}^{2}+\theta_{1}^{2}+\theta_{t}^{2}\right) / 2, A_{1}=\left(\Theta_{\infty}^{2}-\theta_{0}^{2}\right)\left(\theta_{t}^{2}-\theta_{1}^{2}\right) / 4, A_{2}=\left(\Theta_{\infty}^{2}-\theta_{t}^{2}\right)\left(\theta_{1}^{2}-\theta_{0}^{2}\right) / 4, \\
& A_{3}=\left(\Theta_{\infty}^{2}-\theta_{1}^{2}\right)\left(\theta_{0}^{2}-\theta_{t}^{2}\right) / 4, A_{4}=\left\{\left(\Theta_{\infty}^{2}+\theta_{t}^{2}\right)\left(\theta_{0}^{2}-\theta_{1}^{2}\right)^{2}+\left(\Theta_{\infty}^{2}-\theta_{t}^{2}\right)\left(\theta_{0}^{2}+\theta_{1}^{2}\right)\right\} / 32, \\
& B_{3}=A_{3}+A_{0}^{2} / 4 .
\end{aligned}
$$

F-VII $\left(-\frac{1}{4}-2 \beta_{1} / \hbar^{2}, 0\right)$ is reduced to the sixth Painlevé equation with $\theta_{1}^{2}=\theta_{0}^{2}$ because of the symmetry of F-VII $\left(A_{0}, A_{2}\right)$.

\subsection{Degeneration Scheme of the Painlevé Equations}

Six Painlevé equations are the nonlinear ODEs which define the special functions containing Gauss' hypergeometric function, Bessel functions, Airy functions, etc., and yields elliptic functions and trigonometric functions as the autonomous limits [5] [6]. Solutions to the Painlevé equations are called the Painlevé transcendents. So, the Painlevé transcendents are the ancestors of all classical special functions satisfying ODEs. And, all of the Painlevé equations are derived from the sixth Painlevé equation by some limitation which is called the degeneration scheme [5] [7]. For example, the fifth Painlevé equation:

$$
\begin{aligned}
u^{\prime \prime}= & \{1 / 2 u+1 /(u-1)\} u^{\prime 2}-u^{\prime} / t+\left\{(u-1)^{2} / t^{2}\right\}\left[\left(\theta_{\infty}^{2} / 2\right) u+\left(-\theta_{0}^{2} / 2\right) / u\right] \\
& +\eta(1+\kappa) u / t+\left(-\eta^{2} / 2\right) u(u+1) /(u-1)
\end{aligned}
$$

is derived from the sixth Painlevé equation as follows: replace $\left(u, t ; \theta_{\infty}, \theta_{0}, \theta_{1}, \theta_{t}\right)$ by $\left(u, 1+\varepsilon t ; \theta_{\infty}, \theta_{0}, \eta / \varepsilon+\kappa,-\eta / \varepsilon\right)$, and then take limitation $\varepsilon \rightarrow 0$.

In this article, we lift-up the degeneration scheme of the Painlevé equation to the superintegrable system, and get the system with potential described by the fifth Painlevé transcendents. The degenerated system should break one or more rules for classification set up by Tremblay and Winternitz [1].

\section{Results}

Theorem 1. By change of variables $t=s /(s-1), w=\left(v+\frac{1}{4} A_{0} s\right) /(1-s)$, the superintegrable system obtained by Tremblay and Winternitz [1] is reduced into the system

$$
\begin{aligned}
& H=-\frac{\hbar^{2}}{2}\left[\partial_{r}^{2}+r^{-1} \partial_{r}+r^{-2}\left(\sqrt{-s}(s-1) \partial_{s}\right)^{2}\right]+S(s) / r^{2}, \\
& X=-\hbar^{2}\left(\sqrt{-s}(s-1) \partial_{s}\right)^{2}+2 S(s), \\
& Y /(-i \hbar)=-\hbar^{2}\left\{\left(\sqrt{-s}(s-1) \partial_{s}\right)^{2}, \partial_{x 1}\right\}+\left\{g_{1}, \partial_{x 1}\right\}+\left\{g_{2}, \partial_{x 2}\right\},
\end{aligned}
$$

where $S=\sqrt{-s}(s-1) \partial_{s} T, \partial_{\theta}=\sqrt{-s}(s-1) \partial_{s}, \quad \partial_{x 1}=\frac{1+s}{1-s} \partial_{r}-\frac{2 s}{r} \partial_{s} \quad \partial_{x 2}=\frac{2 \sqrt{-s}}{1-s} \partial_{r}-\frac{\sqrt{-s}(1+s)}{r} \partial_{s}$.

Moreover, F-VII $\left(A_{0}, 0\right)$ is reduced to F-VII $\left(A_{0},-A_{0}^{2} / 4\right)$, i.e. if $w=w(t)$ solves F-VII $\left(A_{0}, 0\right)$, then $v=v(s)$ solves F-VII $\left(A_{0},-A_{0}^{2} / 4\right)$.

Theorem 2. By the degeneration scheme from the six Painlevé equation to the fifth Painlevé equation, the system is reduced into the one

$$
\begin{aligned}
H & =-\frac{\hbar^{2}}{2}\left[\partial_{r}^{2}+r^{-1} \partial_{r}+r^{-2}\left(i s \partial_{s}\right)^{2}\right]+S(s) / r^{2}, \\
X & =-\hbar^{2}\left(i s \partial_{s}\right)^{2}+2 S(s),
\end{aligned}
$$




$$
Y /(-i \hbar)=-\hbar^{2}\left\{\left(i s \partial_{s}\right)^{2}, \partial_{x 1}\right\}+\left\{g_{1}, \partial_{x 1}\right\}+\left\{g_{2}, \partial_{x 2}\right\}
$$

where $S=i s \partial_{s} T, \partial_{\theta}=i s \partial_{s}, \partial_{x 1}=-\frac{2}{s} \partial_{r}-\frac{2}{r} \partial_{s} \quad \partial_{x 2}=-\frac{2 i}{s} \partial_{r}-\frac{2 i}{r} \partial_{s}$.

Theorem 3. By change of the independent variable $s=\exp (i \sigma)$ and $(x, y)=(r \cos \sigma, r \sin \sigma)$, the system is reduced into the one

$$
\begin{aligned}
& H=-\frac{\hbar^{2}}{2}\left[\partial_{r}^{2}+r^{-1} \partial_{r}+r^{-2} \partial_{\sigma}^{2}\right]+S(\sigma) / r^{2}, \\
& X=-\hbar^{2} \partial_{\sigma}^{2}+2 S(\sigma), \\
& Y /(-i \hbar)=-\hbar^{2}\left\{\partial_{\sigma}^{2}, \partial_{x 1}\right\}+\left\{g_{1}, \partial_{x 1}\right\}+\left\{g_{2}, \partial_{x 2}\right\},
\end{aligned}
$$

where $S=\partial_{\sigma} T, \partial_{\sigma}=i s \partial_{s}, \partial_{x 1}=-2 \partial_{x}+2 i \partial_{y}, \partial_{x 2}=-2 \partial_{y}-2 i \partial_{x}$.

Each theorem is obtained by a straight-forward computation.

\section{Discussion}

The degeneration scheme broke the reality of the coordinates, which is not a surprising conclusion. The fact says that, if the assumption of the Tremblay and Winternitz [1] is made looser, then another superintegrable system may appear. So, the author thinks that the assumption of the Hermitian nature is too strong to get the sixth Painlevé equation with full-parameter or other Painlevé equations.

Marquette and Winternitz [8] also obtained other superintegrable systems with potentials described by the first, second and fourth Painlevé equations. But it is uncertain if the system above degenerates into the system obtained in [8].

\section{Acknowledgements}

The author thanks Professor R. Conte for introducing him the superintegrable systems obtained by Winternitz school, and for thoughtfulness in his stay at Ecole Normale Supérieure de Cachan.

This research is partially supported by "Strategic Fostering Program for Young Researchers Engaged in Natural Sciences toward the Establishment of the Sustainable Society” of Hiroshima University funded by "Institutional Program for Young Researcher Overseas Visits” of Japan Society for the Promotion of Science.

\section{References}

[1] Tremblay, F. and Winternitz, P. (2010) Third-Order Superintegrable Systems Separating in Polar Coordinates. Journal of Physics A: Mathematical and Theoretical, 43, Article ID: 175206 (17p). http://dx.doi.org/10.1088/1751-8113/43/17/175206

[2] Cosgrove, C.M. (2006) Higher Order Painlevé Equations in the Polynomial Class II. Bureau Symbol P1. Studies in Applied Mathematics, 116, 321-413. http://dx.doi.org/10.1111/j.1467-9590.2006.00346.x

[3] Cosgrove, C.M. and Scoufis, G. (1993) Painlevé Classification of a Class of Differential Equations of the Second Order and Second Degree. Stud. Appl. Math., 88, 25-87.

[4] Conte, R., Grundland, A.M. and Musette, M. (2006) A Reduction of the Resonant Three-Wave Interaction to the Generic Sixth Painlevé Equation. Journal of Physics A: Mathematical and General, 39, 12115-12127. http://dx.doi.org/10.1088/0305-4470/39/39/S07

[5] Conte, R. and Musette, M. (2008) The Painlevé Handbook. Springer Science+Business Media B.V., Dordrecht.

[6] Ince, E.L. (1956) Ordinary Differential Equations. Dover Publ., Inc., New York.

[7] Okamoto, K. (1986) Isomonodromic Deformation and Painlevé Equations, and the Garnier System. J. Fac. Sci. Univ. Tokyo, Sect. IA, 33, 575-618.

[8] Marquette, I. and Winternitz, P. (2008) Superintegrable Systems with Third-Order Integrals of Motion. Journal of Physics A: Mathematical and General, 41, Article ID: 304031 (10p). http://dx.doi.org/10.1088/1751-8113/41/30/304031 